\title{
Discontinuous Galerkin methods for the Stokes equations using divergence-free approximations
}

\author{
A. Montlaur ${ }^{1,2}$, S. Fernandez-Mendez ${ }^{1,3}$ and A. Huerta ${ }^{1,3, *, \dagger}$ \\ ${ }^{1}$ Laboratori de Càlcul Numèric (LaCàN), Universitat Politècnica de Catalunya, Jordi Girona 1-3, \\ 08034 Barcelona, Spain \\ ${ }^{2}$ Escola Politècnica Superior de Castelldefels, Universitat Politècnica de Catalunya, Castelldefels, Spain \\ ${ }^{3}$ Departament de Matemàtica Aplicada III, E.T.S. Ingenieros de Caminos, Universitat Politècnica de Catalunya, \\ Jordi Girona 1, E-08034 Barcelona, Spain
}

\begin{abstract}
SUMMARY
A discontinuous Galerkin (DG) method with solenoidal approximation for the simulation of incompressible flow is proposed. It is applied to the solution of the Stokes equations. The interior penalty method is employed to construct the DG weak form. For every element, the approximation space for the velocity field is decomposed as the direct sum of a solenoidal space and an irrotational space. This allows to split the DG weak form into two uncoupled problems: the first one solves for the velocity and the hybrid pressure (pressure along the mesh edges) and the second one allows the computation of the pressure in the element interior. Furthermore, the introduction of an extra penalty term leads to an alternative DG formulation for the computation of solenoidal velocities with no presence of pressure terms. Pressure can then be computed as a post-process of the velocity solution. Numerical examples demonstrate the applicability of the proposed methodologies.
\end{abstract}

KEY WORDS: discontinuous Galerkin; Stokes equations; incompressible flow; divergence-free; solenoidal; interior penalty method

\section{INTRODUCTION}

Research in finite element methods for the numerical solution of problems with incompressibility constraints has been very active in the past decades. These problems have a large number of applications ranging from the simulation of incompressible fluids to the solution of Maxwell's equations

\footnotetext{
*Correspondence to: A. Huerta, Departament de Matemàtica Aplicada III, E.T.S. Ingenieros de Caminos, Universitat Politècnica de Catalunya, Jordi Girona 1, E-08034 Barcelona, Spain.

†E-mail: antonio.huerta@upc.edu,www-lacan.upc.edu

Contract/grant sponsor: Ministerio de Educación y Ciencia; contract/grant numbers: BIA2007-66965, DPI2007-62395

Contract/grant sponsor: Generalitat de Catalunya AGAUR; contract/grant number: 2005SGR917
} 
in electrodynamic problems. An interesting alternative is to use explicit divergence-free bases in order to solve problems with incompressibility. Crouzeix and Raviart [1] were the first to construct divergence-free elements in order to eliminate the pressure in the final equation. They used triangular conforming and non-conforming elements where the incompressibility condition was only approximately satisfied. Griffiths [2] proposed an element-level divergence-free basis for several finite element schemes on triangular and quadrilateral elements. Nevertheless, a major limitation of these techniques is that continuous and weakly divergence-free (or discretely divergence-free following the notation of [3]) approximation spaces are difficult to generalize for higher-order approximations.

More recently, several authors have focused their attention on discontinuous Galerkin (DG) formulations for computational fluid dynamics [4] and in particular for the Stokes equations [5-7]. The attractiveness of DG method is mainly due to its stability properties in convectiondominated problems, its efficiency for high-order computations, which allows $h p$-adaptive refinement, and local conservation properties. Moreover, in a DG framework, divergence-free high-order approximations can be easily defined: an element-by-element discontinuous approximation with a divergence-free polynomial base in each element can be considered with a straightforward definition for high-order approximations [5,6]. Because of the important costs of DG methods, the reduction in degrees of freedom (both in velocity and pressure) induced by a divergence-free approach is very interesting from a computational point of view.

In the 1990s, Baker et al. [5] and Karakashian and Jureidini [8] developed and analyzed a DG formulation with a piecewise polynomial divergence-free velocity, with optimal error bounds. Nevertheless, this formulation has some limitations: it requires the use of continuous pressure approximations; only Dirichlet boundary conditions are considered (in fact, natural boundary conditions cannot be easily imposed), and different computational meshes (with different mesh sizes) must be considered for velocity and pressure to ensure stability.

A DG method for the Stokes equations with piecewise polynomial approximations was also proposed and analyzed by Toselli [7], but without the pointwise imposition of the divergencefree condition. This DG formulation shows better stability properties than continuous Galerkin approximations, and uniform divergence stability is proven when velocity is approximated one or two degrees higher than pressure. In fact, equal-order interpolation numerical results show no spurious pressure modes although no uniform stability properties are proven. Unfortunately, the bilinear form related with velocities is non-symmetric, and the DG advantages for the definition of piecewise solenoidal approximations are not exploited.

More recently, Cockburn and coworkers propose $[6,9,10]$ a DG formulation with solenoidal piecewise polynomial approximations. It is derived from a local discontinuous Galerkin (LDG) rationale based on a mixed formulation of the problem (with velocity, vorticity and pressure), and with the introduction of numerical traces. The concept of hybrid pressures is also introduced, that is, pressures along the element sides. Pressures in the interior of the elements are computed as a post-process of the LDG solution. For analysis purposes, the LDG formulation is expressed in compact form in [9]. With the introduction of proper lifting operators, the vorticity is replaced in the LDG formulation leading to a velocity-pressure formulation with symmetric and coercive bilinear form for velocities.

In this work, a new DG formulation with piecewise solenoidal polynomial velocity and hybrid pressures is proposed. It is derived from an interior penalty method (IPM) rationale [11, 12], leading also to a symmetric and coercive bilinear form for velocities. As for the LDG formulation, the approximation space for the velocity field is decomposed in every element as direct sum of 
solenoidal and irrotational polynomial spaces. This also allows to split the IPM weak form into two uncoupled problems: the first one solves for velocity and hybrid pressure and the second one allows the evaluation of pressures in the interior of the elements. The resulting method has many points in common with the LDG formulation in compact form stated in [9]. Namely, both are formulated in terms of piecewise solenoidal velocities and hybrid pressures, the bilinear form is symmetric and positive definite, and the pressure in the interior of the elements is computed as a post-process of the solution. Nevertheless, different rationales are followed for the LDG and IPM methods, leading to completely different formulations. For instance, one of the most remarkable differences is that the IPM formulation proposed here does not involve lifting operators that induce an approximate orthogonality property in the LDG formulation [9].

The IPM weak problem is also reformulated as a minimization problem subject to the constraint of normal continuity of the velocity field. The solution of this optimization problem with the introduction of a non-consistent penalty leads to an alternative DG formulation for the computation of solenoidal velocities with no presence of pressure terms (i.e. solving a system with symmetric positive-definite matrix). Pressure can then be computed as a post-process of the velocity solution. This second IPM method exactly coincides with the DG method proposed in [13], where different alternatives for the approximation, based on the definition of a piecewise continuous stream function spaces, are also proposed and analyzed. In fact, it is worth noting the contributions in solid mechanics by Hansbo and co-workers $[12,14,15]$, which have inspired several authors (see, for instance, [16] for the solution of the Navier-Stokes equations) and in particular this paper.

The contributions of this paper are presented as follows. The derivation of a new DG IPM formulation for the solution of Stokes problems, with Dirichlet and Neumann boundary conditions, is presented in detail in Section 3.1. The particularization of the IPM weak form with a splitting of the velocity space in solenoidal and irrotational parts is presented and analyzed in Section 3.2. In Section 3.3, the DG method initially proposed by Hansbo and Larson in [13] is presented with an alternative derivation. The implementation of Neumann boundary conditions is included in the formulation, and a methodology for the computation of pressures as a post-process of the velocity solution is proposed. Numerical tests demonstrate the applicability of both methodologies (IPM and IPM with non-consistent penalty) for the solution of the Stokes equations in Section 5. The selection of the penalization parameters in order to achieve optimal convergence rates is also studied. Finally, the IPM formulation is used for the simulation of a fluid flow through a porous medium.

\section{THE STOKES PROBLEM}

Let $\Omega \subset \mathbb{R}^{n_{\mathrm{sd}}}$ be an open bounded domain with piecewise linear boundary $\partial \Omega$ and $\mathrm{n}_{\mathrm{sd}}$ the number of spatial dimensions. Suppose that $\Omega$ is partitioned in $n_{e l}$ disjoint subdomains $\Omega_{i}$, which for example correspond to different materials, with also piecewise linear boundaries $\partial \Omega_{i}$ which define an internal interphase $\Gamma$; the following definitions and notations are used:

$$
\begin{gathered}
\bar{\Omega}=\bigcup_{i=1}^{\mathrm{n}_{\mathrm{e} 1}} \bar{\Omega}_{i}, \quad \Omega_{i} \cap \Omega_{j}=\emptyset \quad \text { for } i \neq j \\
\widehat{\Omega}:=\bigcup_{i=1}^{\mathrm{n}_{\mathrm{e} 1}} \Omega_{i} \quad \text { and } \quad \Gamma:=\bigcup_{\substack{i, j=1 \\
i \neq j}}^{\mathrm{n}_{\mathrm{e} 1}} \bar{\Omega}_{i} \cap \bar{\Omega}_{j}=\left[\bigcup_{i=1}^{\mathrm{n}_{\mathrm{e}}} \partial \Omega_{i}\right] \backslash \partial \Omega
\end{gathered}
$$


The strong form of the homogeneous Stokes problem can be expressed as

$$
\begin{aligned}
& -\nabla \cdot \sigma=\mathbf{s} \text { in } \widehat{\Omega} \\
& \boldsymbol{\nabla} \cdot \mathbf{u}=0 \quad \text { in } \widehat{\Omega} \\
& \mathbf{u}=\mathbf{u}_{\mathrm{D}} \text { on } \Gamma_{\mathrm{D}} \\
& \mathbf{n} \cdot \boldsymbol{\sigma}=\mathbf{t} \quad \text { on } \Gamma_{\mathrm{N}} \\
& \llbracket \mathbf{n} \otimes \mathbf{u} \rrbracket=\mathbf{0} \quad \text { on } \Gamma \\
& \llbracket \mathbf{n} \cdot \boldsymbol{\sigma} \rrbracket=\mathbf{0} \quad \text { on } \Gamma
\end{aligned}
$$

where $\partial \Omega=\bar{\Gamma}_{\mathrm{D}} \cup \bar{\Gamma}_{\mathrm{N}}, \quad \Gamma_{\mathrm{D}} \cap \Gamma_{\mathrm{N}}=\emptyset, \quad \mathbf{s} \in \mathcal{L}_{2}(\Omega)$ is a source term, $\boldsymbol{\sigma}$ is the ('dynamic' or 'density-scaled') Cauchy stress, which is related to velocity, $\mathbf{u}$, and pressure, $p$, by the linear Stokes' law

$$
\boldsymbol{\sigma}=-p \mathbf{I}+2 v \nabla^{\mathrm{S}} \mathbf{u}
$$

with $v$ being the kinematic viscosity and $\nabla^{\mathrm{S}}=\frac{1}{2}\left(\nabla+\nabla^{\mathrm{T}}\right)$.

The jump $\llbracket \cdot \rrbracket$ and the mean $\{\cdot\}$ operators are defined along the interface $\Gamma$ using values from the elements to the left and right of the interface - say, $\Omega_{i}$ and $\Omega_{j}$ —and are also extended along the exterior boundary—only values in the interior of $\Omega$ are employed—namely

$$
\llbracket \odot \rrbracket=\left\{\begin{array}{ll}
\bigcirc i+\bigcirc j & \text { on } \Gamma \\
\bigcirc & \text { on } \partial \Omega
\end{array} \quad \text { and } \quad\{\bigcirc\}= \begin{cases}\kappa_{i} \bigcirc_{i}+\kappa_{j} \bigcirc_{j} & \text { on } \Gamma \\
\bigcirc & \text { on } \partial \Omega\end{cases}\right.
$$

Usually $\kappa_{i}=\kappa_{j}=\frac{1}{2}$ but, in general, these two scalars are only required to verify $\kappa_{i}+\kappa_{j}=1$, see, for instance, [12]. Note that definitions such as

$$
\kappa_{i}= \begin{cases}1 & \text { if } \Omega_{i} \text { is the largest } \\ 0 & \text { otherwise }\end{cases}
$$

are also possible.

The major difference between the mean and the jump operator is that the latter always involves the normal to the interface or to the domain. Given two contiguous subdomains $\Omega_{i}$ and $\Omega_{j}$, their exterior unit normals are denoted by, respectively, $\mathbf{n}_{i}$ and $\mathbf{n}_{j}$ (recall that $\mathbf{n}_{i}=-\mathbf{n}_{j}$ ) and along $\partial \Omega$ the exterior unit normal is denoted by $\mathbf{n}$. In what follows, the jump operator as defined previously will appear in these three cases:

$$
\begin{aligned}
& \llbracket p \mathbf{n} \rrbracket=\left\{\begin{array}{lll}
p_{i} \mathbf{n}_{i}+p_{j} \mathbf{n}_{j}=\mathbf{n}_{i}\left(p_{i}-p_{j}\right) & \text { on } \Gamma \\
p \mathbf{n} & \text { on } \partial \Omega & \text { for scalars }
\end{array}\right. \\
& \llbracket \mathbf{n} \otimes \mathbf{v} \rrbracket=\left\{\begin{array}{lll}
\mathbf{n}_{i} \otimes \mathbf{v}_{i}+\mathbf{n}_{j} \otimes \mathbf{v}_{j}=\mathbf{n}_{i} \otimes\left(\mathbf{v}_{i}-\mathbf{v}_{j}\right) & \text { on } \Gamma \\
\mathbf{n} \otimes \mathbf{v} & \text { on } \partial \Omega & \text { or }
\end{array}\right.
\end{aligned}
$$


DG METHODS FOR THE STOKES EQUATIONS

$$
\begin{array}{r}
\llbracket \mathbf{n} \cdot \mathbf{v} \rrbracket=\left\{\begin{array}{ll}
\mathbf{n}_{i} \cdot \mathbf{v}_{i}+\mathbf{n}_{j} \cdot \mathbf{v}_{j}=\mathbf{n}_{i} \cdot\left(\mathbf{v}_{i}-\mathbf{v}_{j}\right) & \text { on } \Gamma \\
\mathbf{n} \cdot \mathbf{v} & \text { on } \partial \Omega
\end{array}\right. \text { for vectors } \\
\llbracket \mathbf{n} \cdot \boldsymbol{\sigma} \rrbracket=\left\{\begin{array}{ll}
\mathbf{n}_{i} \cdot \boldsymbol{\sigma}_{i}+\mathbf{n}_{j} \cdot \boldsymbol{\sigma}_{j}=\mathbf{n}_{i} \cdot\left(\boldsymbol{\sigma}_{i}-\boldsymbol{\sigma}_{j}\right) & \text { on } \Gamma \\
\mathbf{n} \cdot \boldsymbol{\sigma} & \text { on } \partial \Omega
\end{array}\right. \text { for second-order tensors }
\end{array}
$$

This definition of the jump was previously considered by other authors, see, for instance, [9], and presents two important advantages: first, it does not depend on a selection of a privileged normal sign on the edges in $2 \mathrm{D}$ or faces in $3 \mathrm{D}$, and second, the input and output spaces for the operator coincide, that is, the jump of a scalar is a scalar, the jump of a vector is a vector, etc. Other definitions have been more popular in the past, but do not have these advantages. For instance, the jump at an edge $\Gamma_{\mathrm{E}}$, shared by two elements $\Omega_{i}$ and $\Omega_{j}$ with $i<j$, could be defined as $\llbracket u \rrbracket=u_{i}-u_{j}$, see [5] among others. This definition involves the decision of a privileged normal sign; therefore, it may lead to weak definitions with a not desirable dependency on this choice. Another alternative definition would be $\llbracket u \rrbracket=u_{i} \mathbf{n}_{i}+u_{j} \mathbf{n}_{j}$ for scalar $u, \llbracket \mathbf{u} \rrbracket=\mathbf{u}_{i} \cdot \mathbf{n}_{i}+\mathbf{u}_{j} \cdot \mathbf{n}_{j}$ for vector $\mathbf{u}$, etc., see, for instance, [17]. It also does not require the selection of a normal sign, but it has different spaces for the input and the output: the jump of a scalar is a vector and the jump of a vector is a scalar. Moreover, the use of this definition camouflages the presence of the normal in the weak formulation: note that the evaluation of $\llbracket u \rrbracket$ involves the normal, although the normal does not explicitly appear in the weak form. Thus, in the authors' opinion the jump operator (3) leads to more easily readable weak formulations. Nevertheless, there is one situation where jump (3) or the definition used in [17] present some limitations: the computation of the jump of a scalar function with no presence of the normal vector. In the following, this computation appears only for terms of the form $\left(\mathbf{u}_{i}-\mathbf{u}_{j}, \mathbf{v}_{i}-\mathbf{v}_{j}\right)_{\Gamma_{\mathrm{E}}}$, where $\Omega_{i}$ and $\Omega_{j}$ are the elements sharing the interface $\Gamma_{\mathrm{E}}$, and the following identity is used:

$$
\left(\mathbf{u}_{i}-\mathbf{u}_{j}, \mathbf{v}_{i}-\mathbf{v}_{j}\right)_{\Gamma_{\mathrm{E}}}=(\llbracket \mathbf{n} \otimes \mathbf{u} \rrbracket, \llbracket \mathbf{n} \otimes \mathbf{v} \rrbracket)_{\Gamma_{\mathrm{E}}}
$$

\section{THE WEAK FORM OF THE STOKES PROBLEM}

Following the usual methodology in the DG framework, the weak problem from the strong form defined by (1) is considered for each domain $\Omega_{i}$. That is, find $\mathbf{u}_{i} \in\left[\mathcal{H}^{1}\left(\Omega_{i}\right)\right]^{\mathrm{n}_{\mathrm{sd}}}$ and $p_{i} \in \mathcal{L}_{2}\left(\Omega_{i}\right)$ for $i=1, \ldots, \mathrm{n}_{\mathrm{el}}$, which comply the boundary conditions (1c), (1e) and (1f) such that

$$
a_{\Omega_{i}}\left(\mathbf{u}_{i}, \mathbf{v}\right)+b_{\Omega_{i}}\left(\mathbf{v}, p_{i}\right)-\left(\mathbf{n}_{i} \cdot \boldsymbol{\sigma}\left(\mathbf{u}_{i}, p_{i}\right), \mathbf{v}\right)_{\partial \Omega_{i} \backslash \Gamma_{\mathrm{N}}}+b_{\Omega_{i}}\left(\mathbf{u}_{i}, q\right)=l_{\Omega_{i}}(\mathbf{v})+(\mathbf{t}, \mathbf{v})_{\partial \Omega_{i} \cap \Gamma_{\mathrm{N}}}
$$

for all $(\mathbf{v}, q) \in\left[\mathcal{H}^{1}\left(\Omega_{i}\right)\right]^{\mathrm{n}_{\mathrm{sd}}} \times \mathcal{L}_{2}\left(\Omega_{i}\right)$, where

$$
\begin{gathered}
a_{\Omega_{i}}(\mathbf{v}, \mathbf{w})=\int_{\Omega_{i}} 2 v \nabla^{\mathrm{S}} \mathbf{v}: \nabla^{\mathrm{S}} \mathbf{w} \mathrm{d} \Omega, \quad b_{\Omega_{i}}(\mathbf{v}, q)=-\int_{\Omega_{i}} q \nabla \cdot \mathbf{v} \mathrm{d} \Omega \\
l_{\Omega_{i}}(\mathbf{v})=\int_{\Omega_{i}} \mathbf{s v} \mathrm{d} \Omega
\end{gathered}
$$


In the previous and the following equations, $(\cdot, \cdot)_{\Upsilon}$ denotes the $\mathcal{L}_{2}$ scalar product in any domain $\Upsilon \subset \Gamma \cup \partial \Omega$, that is

$$
\begin{gathered}
(p, q)_{\Upsilon}=\int_{\Upsilon} p q \mathrm{~d} \Gamma \quad \text { for scalars } \\
(\mathbf{u}, \mathbf{v})_{\Upsilon}=\int_{\Upsilon} \mathbf{u} \cdot \mathbf{v} \mathrm{d} \Gamma \quad \text { for vectors } \\
(\boldsymbol{\sigma}, \tau)_{\Upsilon}=\int_{\Upsilon} \boldsymbol{\sigma}: \tau \mathrm{d} \Gamma \quad \text { for second-order tensors }
\end{gathered}
$$

In order to rewrite all $\mathrm{n}_{\mathrm{el}}$ weak problems defined in (7) as one weak problem, let $\mathbf{u}$ be such that its restriction to $\Omega_{i}$ is $\mathbf{u}_{i}$, namely $\mathbf{u} \in\left[\mathcal{H}^{1}(\widehat{\boldsymbol{\Omega}})\right]^{\mathrm{n}_{\mathrm{sd}}}$ with

$$
\left[\mathcal{H}^{1}(\widehat{)}]^{\mathrm{n}_{\mathrm{sd}}}:=\left\{\mathbf{v} \in\left[\mathcal{L}_{2}(\Omega)\right]^{\mathrm{n}_{\mathrm{sd}}}|\mathbf{v}|_{\Omega_{i}} \in\left[\mathcal{H}^{1}\left(\Omega_{i}\right)\right]^{\mathrm{n}_{\mathrm{sd}}} \text { for } i=1, \ldots, \mathrm{n}_{\mathrm{el}}\right\}\right.
$$

and, similarly, $p \in \mathcal{L}_{2}(\Omega)$ is such that its restriction to $\Omega_{i}$ is $p_{i}$. Differential operators are assumed to act on these functions piecewise and not in the sense of distributions. Thus, adding equations (7) for $i=1, \ldots, \mathrm{n}_{\mathrm{e}}$, the unique weak problem becomes: find $\mathbf{u}$ and $p$ such that

$$
a(\mathbf{u}, \mathbf{v})+b(\mathbf{v}, p)-\sum_{i=1}^{\mathrm{n}_{\mathrm{el}}}\left(\mathbf{n}_{i} \cdot \boldsymbol{\sigma}\left(\mathbf{u}_{i}, p_{i}\right), \mathbf{v}\right)_{\partial \Omega_{i} \backslash \Gamma_{\mathrm{N}}}+b(\mathbf{u}, q)=l(\mathbf{v})
$$

for all test functions $\mathbf{v} \in\left[\mathcal{H}^{1}(\widehat{\Omega})\right]^{\mathrm{n}_{\mathrm{sd}}}$ and $q \in \mathcal{L}_{2}(\Omega)$; where the bilinear forms are now integrated over the whole domain $\Omega$, namely

$$
a(\mathbf{v}, \mathbf{w})=\int_{\Omega} 2 v \nabla^{\mathrm{S}} \mathbf{v}: \nabla^{\mathrm{S}} \mathbf{w} \mathrm{d} \Omega, \quad b(\mathbf{v}, q)=-\int_{\Omega} q \nabla \cdot \mathbf{v} \mathrm{d} \Omega
$$

and

$$
l(\mathbf{v})=\int_{\Omega} \mathbf{s v} \mathrm{d} \Omega+(\mathbf{t}, \mathbf{v})_{\Gamma_{\mathrm{N}}}
$$

For two contiguous subdomains, $\Omega_{i}$ and $\Omega_{j}$, with a common boundary $\Gamma_{\mathrm{e}} \subset \Gamma$ it is easy to check that

$$
\begin{aligned}
& \left(\mathbf{n}_{i} \cdot \boldsymbol{\sigma}\left(\mathbf{u}_{i}, p_{i}\right), \mathbf{v}_{i}\right)_{\Gamma_{\mathrm{e}}}+\left(\mathbf{n}_{j} \cdot \boldsymbol{\sigma}\left(\mathbf{u}_{j}, p_{j}\right), \mathbf{v}_{j}\right)_{\Gamma_{\mathrm{e}}} \\
& \quad=(\{\boldsymbol{\sigma}(\mathbf{u}, p)\}, \llbracket \mathbf{n} \otimes \mathbf{v} \rrbracket) \Gamma_{\Gamma_{\mathrm{e}}}+\left(\llbracket \mathbf{n} \cdot \boldsymbol{\sigma}(\mathbf{u}, p) \rrbracket, \kappa_{j} \mathbf{v}_{i}+\kappa_{i} \mathbf{v}_{j}\right)_{\Gamma_{\mathrm{e}}}
\end{aligned}
$$

Moreover, the boundary condition (1f) simplifies the previous equation because the last term is zero. Thus, from the previous equation the weak form (8) can be rewritten as

$$
a(\mathbf{u}, \mathbf{v}) \quad b(\mathbf{v}, p)-(\{\boldsymbol{\sigma}(\mathbf{u}, p)\}, \llbracket \mathbf{n} \otimes \mathbf{v} \rrbracket)_{\Gamma}-(\mathbf{n} \cdot \boldsymbol{\sigma}(\mathbf{u}, p), \mathbf{v})_{\Gamma_{\mathrm{D}}}+b(\mathbf{u}, q)=l(\mathbf{v})
$$

This expression can be further simplified using the extension of the jump and mean operators on the exterior boundary, in particular, in this case along $\Gamma_{\mathrm{D}}$, and the identity $\mathbf{n} \cdot \boldsymbol{\sigma} \cdot \mathbf{v}=\boldsymbol{\sigma}:(\mathbf{n} \otimes \mathbf{v})$. The weak problem equivalent to (1) becomes: find $\mathbf{u} \in\left[\mathcal{H}^{1}(\widehat{\Omega})\right]^{\mathrm{n}_{\mathrm{sd}}}$ and $p \in \mathcal{L}_{2}(\Omega)$ subject to the boundary conditions defined by (1c) and (1e) such that

$$
a(\mathbf{u}, \mathbf{v})+b(\mathbf{v}, p)-(\{\boldsymbol{\sigma}(\mathbf{u}, p)\}, \llbracket \mathbf{n} \otimes \mathbf{v} \rrbracket)_{\Gamma \cup \Gamma_{\mathrm{D}}} \quad b(\mathbf{u}, q)=l(\mathbf{v})
$$

for all test functions $\mathbf{v} \in\left[\mathcal{H}^{1}(\widehat{\Omega})\right]^{\mathrm{n}_{\mathrm{s}} \mathrm{a}}$ and $q \in \mathcal{L}_{2}(\Omega)$. 


\subsection{The IPM formulation}

Following the standard approach of IPM [11], the previous weak problem (9) is symmetrized and a new term is added to ensure a coercive bilinear form for the velocity. In this process, the boundary conditions (1c) and (1e) — not yet imposed-are used in order to maintain the consistency of the weak problem (i.e. the solution of (1) is the solution of the weak problem). The resulting IPM weak problem can then be expressed as: find $\mathbf{u} \in\left[\mathcal{H}^{1}(\widehat{\Omega})\right]^{\mathrm{n}_{\mathrm{sd}}}$ and $p \in \mathcal{L}_{2}(\Omega)$ such that

$$
\begin{aligned}
& a(\mathbf{u}, \mathbf{v})+b(\mathbf{v}, p)+b(\mathbf{u}, q)-(\{\boldsymbol{\sigma}(\mathbf{u}, p)\}, \llbracket \mathbf{n} \otimes \mathbf{v} \rrbracket) \Gamma \cup \Gamma_{\mathrm{D}}-(\llbracket \mathbf{n} \otimes \mathbf{u} \rrbracket,\{\boldsymbol{\sigma}(\mathbf{v}, q)\})_{\Gamma \cup \Gamma_{\mathrm{D}}} \\
& \quad+\gamma\left(l_{\mathrm{e}}^{-1} \llbracket \mathbf{n} \otimes \mathbf{u} \rrbracket, \llbracket \mathbf{n} \otimes \mathbf{v} \rrbracket\right) \Gamma \cup \Gamma_{\mathrm{D}}=l(\mathbf{v})-\left(\mathbf{u}_{\mathrm{D}}, \mathbf{n} \cdot \boldsymbol{\sigma}(\mathbf{v}, q)\right)_{\Gamma_{\mathrm{D}}}+\gamma\left(l_{\mathrm{e}}^{-1} \mathbf{u}_{\mathrm{D}}, \mathbf{v}\right)_{\Gamma_{\mathrm{D}}}
\end{aligned}
$$

for all $\mathbf{v} \in\left[\mathcal{H}^{1}(\widehat{\Omega})\right]^{\mathrm{n}_{\mathrm{sd}}}$ and $q \in \mathcal{L}_{2}(\Omega)$, where $l_{\mathrm{e}}$ is a measure of each interface $\Gamma_{\mathrm{e}}$ (edge in $2 \mathrm{D}$, face in 3D) and $\gamma$ is a scalar parameter, which must be sufficiently large (to ensure coercivity of the form $a_{\mathrm{IP}}(\cdot, \cdot)$ defined below, see Remark 1). Note that boundary conditions (1c) and (1e) are no longer explicitly mentioned because they are now imposed in weak form.

Using the constitutive law (2) in the previous equation, the weak problem, which presents a symmetric structure, can be expressed as: find $\mathbf{u} \in\left[\mathcal{H}^{1}(\widehat{\Omega})\right]^{\mathrm{n}_{\mathrm{sd}}}$ and $p \in \mathcal{L}_{2}(\Omega)$ such that

$$
\begin{aligned}
& a_{\text {IP }}(\mathbf{u}, \mathbf{v})+b(\mathbf{v}, p)+(\{p\}, \llbracket \mathbf{n} \cdot \mathbf{v} \rrbracket) \Gamma \cup \Gamma_{\mathrm{D}} \\
& \quad+b(\mathbf{u}, q)+(\{q\}, \llbracket \mathbf{n} \cdot \mathbf{u} \rrbracket))_{\Gamma \cup \Gamma_{\mathrm{D}}}=l_{\text {IP }}(\mathbf{v})+\left(q, \mathbf{n} \cdot \mathbf{u}_{\mathrm{D}}\right)_{\Gamma_{\mathrm{D}}}
\end{aligned}
$$

for all $\mathbf{v} \in\left[\mathcal{H}^{1}(\widehat{\Omega})\right]^{\mathrm{n}_{\mathrm{sd}}}$ and $q \in \mathcal{L}_{2}(\Omega)$, with

$$
\begin{aligned}
a_{\mathrm{IP}}(\mathbf{u}, \mathbf{v}):= & a(\mathbf{u}, \mathbf{v})-\left(2 v\left\{\nabla^{\mathrm{S}} \mathbf{u}\right\}, \llbracket \mathbf{n} \otimes \mathbf{v} \rrbracket\right)_{\Gamma \cup \Gamma_{\mathrm{D}}} \\
& -\left(\llbracket \mathbf{n} \otimes \mathbf{u} \rrbracket, 2 v\left\{\nabla^{\mathrm{S}} \mathbf{v}\right\}\right)_{\Gamma \cup \Gamma_{\mathrm{D}}}+\gamma\left(l_{\mathrm{e}}^{-1} \llbracket \mathbf{n} \otimes \mathbf{u} \rrbracket, \llbracket \mathbf{n} \otimes \mathbf{v} \rrbracket\right)_{\Gamma \cup \Gamma_{\mathrm{D}}}
\end{aligned}
$$

and

$$
l_{\text {IP }}(\mathbf{v}):=l(\mathbf{v})-\left(\mathbf{u}_{\mathrm{D}}, 2 v \mathbf{n} \cdot \nabla^{\mathrm{S}} \mathbf{v}\right)_{\Gamma_{\mathrm{D}}}+\gamma\left(l_{\mathrm{e}}^{-1} \mathbf{u}_{\mathrm{D}}, \mathbf{v}\right)_{\Gamma_{\mathrm{D}}}
$$

This weak form is close to the formulation proposed in [7] where stability is also studied. It clearly identifies pressure with the Lagrange multiplier that imposes both a weakly solenoidal field inside each element and a continuous normal component along $\Gamma$. However, the IPM provides a symmetric bilinear form for the velocity, see Equation (11a), whereas the formulation proposed in [7] does not.

An alternative IPM formulation that does not require the evaluation of the divergence of the velocity field can also be obtained from (10). The divergence term is replaced using the following identity valid for any $\mathbf{v} \in\left[\mathcal{H}^{1}(\widehat{\Omega})\right]^{\mathrm{n}_{\mathrm{sd}}}$ and $q \in \mathcal{L}_{2}(\Omega)$ :

$$
b(\mathbf{v}, q)+(\{q\}, \llbracket \mathbf{n} \cdot \mathbf{v} \rrbracket)_{\Gamma \cup \partial \Omega}=(\mathbf{v}, \nabla q)_{\Omega}-\left(\llbracket q \mathbf{n} \rrbracket,\{\{\mathbf{v}\})_{\Gamma}\right.
$$

where the operator $\left\{\{\cdot\}\right.$ is defined at any interior edge $\Gamma_{\mathrm{E}}=\Omega_{i} \cap \Omega_{j}$ as

$$
\left\{\{\mathbf{v}\}=\kappa_{j} \mathbf{v}_{i}+\kappa_{i} \mathbf{v}_{j}\right.
$$

Using this identity and its particularization for $\mathbf{v}=\mathbf{u}$, the solution of the problem (which is continuous and verifies $(1 \mathrm{c}))$, i.e.

$$
b(\mathbf{u}, q)+(q, \mathbf{n} \cdot \mathbf{u})_{\Gamma_{\mathrm{N}}}=(\mathbf{u}, \nabla q)_{\Omega}-\left(\mathbf{n} \cdot \mathbf{u}_{\mathrm{D}}, q\right)_{\Gamma_{\mathrm{D}}}-(\llbracket q \mathbf{n} \rrbracket,\{\{\mathbf{u}\}\})_{\Gamma}
$$


the IPM weak formulation (10) can be expressed as: find $\mathbf{u} \in\left[\mathcal{H}^{1}(\widehat{\Omega})\right]^{\mathrm{n}_{\mathrm{sd}}}$ and $p \in \mathcal{L}_{2}(\Omega)$ such that

$$
\begin{aligned}
& a_{\mathrm{IP}}(\mathbf{u}, \mathbf{v})+(\mathbf{v}, \nabla p)_{\Omega}-(p, \mathbf{n} \cdot \mathbf{v})_{\Gamma_{\mathrm{N}}}-\left(\llbracket p \mathbf{n} \rrbracket,\{\{\mathbf{v}\})_{\Gamma}\right. \\
& \quad+(\mathbf{u}, \nabla q)_{\Omega}-(q, \mathbf{n} \cdot \mathbf{u})_{\Gamma_{\mathrm{N}}}-\left(\llbracket q \mathbf{n} \rrbracket,\{\{\mathbf{u}\})_{\Gamma}=l_{\mathrm{IP}}(\mathbf{v})+\left(\mathbf{n} \cdot \mathbf{u}_{\mathrm{D}}, q\right)_{\Gamma_{\mathrm{D}}}\right.
\end{aligned}
$$

for all $\mathbf{v} \in\left[\mathcal{H}^{1}(\widehat{\Omega})\right]^{\mathrm{n}_{\mathrm{sd}}}$ and $q \in \mathcal{L}_{2}(\Omega)$. The structure of this formulation suggests the use of continuous pressures to simplify the equation, removing the terms with the $\{[\cdot\}\}$ operator. The resulting formulation is more closely related to the work presented in $[5,8]$, where the proposed bilinear form is also symmetric and with no presence of divergence terms. Nevertheless, the weak formulation proposed in $[5,8]$ has some limitations: as commented, it requires the use of continuous approximations for the pressure, it is developed only for Dirichlet boundary conditions and natural boundary conditions cannot be directly imposed, and different computational meshes (with different mesh size) must be considered for velocity and pressure to ensure stability.

In this paper, the IPM formulation (10) is preferred because discontinuous approximations for the pressure are considered and, more importantly, because this weak formulation can be further simplified using piecewise solenoidal approximations.

\subsection{The IPM formulation with solenoidal space}

It is well known that any function in $\left[\mathcal{H}^{1}\left(\Omega_{i}\right)\right]^{\mathrm{n}_{\mathrm{sd}}}$ can be expressed as the sum of a solenoidal part and an irrotational one. Thus, the functional space for the velocity can be split into the direct sum: $\left[\mathcal{H}^{1}(\widehat{\Omega})\right]^{\mathrm{n}} \quad=\mathcal{S} \oplus \mathcal{I}$ where

$$
\begin{aligned}
& \mathcal{S}:=\left\{\mathbf{v} \in\left[\mathcal{H}^{1}(\widehat{\Omega})\right]^{\mathrm{n}_{\mathrm{sd}}}|\nabla \cdot \mathbf{v}|_{\Omega_{i}}=0 \text { for } i=1, \ldots, \mathrm{n}_{\mathrm{el}}\right\} \\
& \mathcal{I} \subset\left\{\mathbf{v} \in\left[\mathcal{H}^{1}(\widehat{\Omega})\right]^{\mathrm{n}_{\mathrm{sd}}}|\nabla \times \mathbf{v}|_{\Omega_{i}}=\mathbf{0} \text { for } i=1, \ldots, \mathrm{n}_{\mathrm{el}}\right\}
\end{aligned}
$$

Note also that $\mathbf{u}$, the solution of the original problem (1) and (10), belongs to $\mathcal{S}$. Under these circumstances, problem (10) can be split into two uncoupled problems, for test functions in $\mathcal{S}$ and $\mathcal{I}$, respectively.

First, divergence-free solution and test functions, $\mathbf{u}, \mathbf{v} \in \mathcal{S}$, are considered in the IPM formulation (10), leading to a simplified IPM formulation with no divergence terms

$$
a_{\mathrm{IP}}(\mathbf{u}, \mathbf{v})+(\{p\}, \llbracket \mathbf{n} \cdot \mathbf{v} \rrbracket) \Gamma_{\Gamma} \Gamma_{\mathrm{D}}+(\{q\}, \llbracket \mathbf{n} \cdot \mathbf{u} \rrbracket){ }_{\Gamma \cup \Gamma_{\mathrm{D}}}=l_{\mathrm{IP}}(\mathbf{v})+\left(q, \mathbf{n} \cdot \mathbf{u}_{\mathrm{D}}\right)_{\Gamma_{\mathrm{D}}}
$$

for all $\mathbf{v} \in \mathcal{S}$ and $q \in \mathcal{L}_{2}(\Omega)$. This formulation is further simplified with the introduction of the space of the so-called hybrid pressures, that is

$$
\mathcal{P}:=\left\{\hat{p} \mid \hat{p}: \Gamma \cup \Gamma_{\mathrm{D}} \longrightarrow \mathbb{R} \text { and } \hat{p}=\llbracket \mathbf{n} \cdot \mathbf{v} \rrbracket \text { for some } \mathbf{v} \in \mathcal{S}\right\}
$$

see [6] for details.

Thus, the first problem for divergence-free velocities and hybrid pressures becomes: find $\mathbf{u} \in \mathcal{S}$ and $\hat{p} \in \mathcal{P}$ such that

$$
\begin{aligned}
& a_{\text {IP }}(\mathbf{u}, \mathbf{v})+(\hat{p}, \llbracket \mathbf{n} \cdot \mathbf{v} \rrbracket) \Gamma_{\Gamma \cup \Gamma_{\mathrm{D}}}=l_{\text {IP }}(\mathbf{v}) \quad \forall \mathbf{v} \in \mathcal{S} \\
& (\hat{q}, \llbracket \mathbf{n} \cdot \mathbf{u} \rrbracket)_{\Gamma \cup \Gamma_{\mathrm{D}}}=\left(\hat{q}, \mathbf{n} \cdot \mathbf{u}_{\mathrm{D}}\right)_{\Gamma_{\mathrm{D}}} \quad \forall \hat{q} \in \mathcal{P}
\end{aligned}
$$


The second problem, which requires the solution of the previous one, i.e. the velocity $\mathbf{u}$ and the hybrid pressure $\hat{p}$, determines the interior pressure: find $p \in \mathcal{L}_{2}(\widehat{\Omega})$

$$
b(\mathbf{v}, p)=l_{\text {IP }}(\mathbf{v})-a_{\text {IP }}(\mathbf{u}, \mathbf{v})-(\hat{p}, \llbracket \mathbf{n} \cdot \mathbf{v} \rrbracket)_{\Gamma \cup \Gamma_{\mathrm{D}}} \quad \forall \mathbf{v} \in \mathcal{I}
$$

Note that this second problem would allow an independent computation of the interior pressure in every domain $\Omega_{i}$.

The IPM formulation with solenoidal and irrotational spaces proposed here has many points in common with the LDG formulation in compact form presented in [9]. Both consider piecewise polynomial approximations, see Section 4, and a splitting of the approximation space as a sum of solenoidal and irrotational parts, leading to two uncoupled problems: the first for velocities and hybrid pressures, and the second for the computation of pressures in the interior of the elements. Moreover, the bilinear form is symmetric, continuous and coercive in both formulations (see Remark 1). Nevertheless, the IPM and the LDG methods correspond to different formulations. In fact, none of the two methods can be expressed as a particular case of the other one. The LDG method is deduced from a mixed formulation of the Stokes problem with velocity, vorticity and pressure, and it is expressed in compact form using proper lifting operators to replace the vorticity. In fact, the presence of lifting operators in the weak form is an important difference with the IPM method, with consequences in the consistency of the formulation. The IPM formulation is a consistent formulation in the sense that the solution of the Stokes problem (1) is also a solution of the IPM weak form, whereas the LDG formulation only verifies an approximate orthogonality property, see [9] for details.

\section{Remark 1}

For $\gamma$ large enough, the IPM bilinear form $a_{\mathrm{IP}}(\cdot, \cdot)$ defined in (11a) is continuous and coercive, that is

$$
a_{\text {IP }}(\mathbf{u}, \mathbf{v}) \leqslant\|\mathbf{u}\||\||\mathbf{v} \|| \quad \forall \mathbf{v} \in \mathcal{S}
$$

and

$$
m\|\mathbf{v}\| \leqslant a_{\text {IP }}(\mathbf{v}, \mathbf{v}) \quad \forall \mathbf{v} \in \mathcal{S}
$$

for some constant $m>0$ independent of the mesh size $h$, where

$$
\|\mathbf{v}\|^{2}=\left\|\nabla^{\mathrm{S}} \mathbf{v}\right\|_{\Omega}^{2}+\left\|h^{1 / 2} \mathbf{n} \cdot\left\{\nabla^{\mathrm{S}} \mathbf{v}\right\}\right\|_{\Gamma \cup \Gamma_{\mathrm{D}}}^{2}+\left\|h^{-1 / 2} \llbracket \mathbf{n} \otimes \mathbf{v} \rrbracket\right\|_{\Gamma \cup \Gamma_{\mathrm{D}}}^{2}
$$

and the $\mathcal{L}^{2}$ norms are defined as

$$
\|\mathbf{f}\|_{\Omega}^{2}=\sum_{i} \int_{\Omega_{i}} \mathbf{f}: \mathbf{f} \mathrm{d} \Omega, \quad\|f\|_{\Gamma \cup \Gamma_{\mathrm{D}}}^{2}=(f, f)_{\Gamma \cup \Gamma_{\mathrm{D}}}
$$

These properties can be proved following standard arguments, see [13,14] for details.

\subsection{IPM formulation with penalization of the discontinuity}

The IPM formulation with solenoidal spaces presented in the previous section, see Equation (14a), allows a computation of the velocity solution involving the pressure only in the boundary of the domains $\Omega_{i}$, i.e. the hybrid pressure. The aim of this section is more ambitious: to obtain a completely decoupled formulation allowing the computation of the solenoidal velocity, but with 
no presence of pressures at all. As proposed in [13], the introduction of a new penalty in the weak formulation achieves this purpose. However, the price of a totally decoupled velocity-pressure formulation is the loss of consistency, which provokes the ill-conditioning typical for non-consistent penalty formulations.

The DG formulation initially proposed and analyzed in [13] is deduced next from an alternative rationale, based on the IPM formulation (14a) and the introduction of a non-consistent penalty. The IPM formulation with solenoidal velocities (14a) can be rewritten as a saddle-point problem, namely

$$
(\mathbf{u}, \hat{p})=\arg \min _{\mathbf{v} \in \mathcal{S}} \max _{\hat{q} \in \mathcal{P}} \frac{1}{2} a_{\text {IP }}(\mathbf{v}, \mathbf{v})-l_{\mathrm{IP}}(\mathbf{v})+(\hat{q}, \llbracket \mathbf{n} \cdot \mathbf{v} \rrbracket) \Gamma_{\Gamma \cup \Gamma_{\mathrm{D}}}-\left(\hat{q}, \mathbf{n} \cdot \mathbf{u}_{\mathrm{D}}\right)_{\Gamma_{\mathrm{D}}}
$$

or, equivalently, as a minimization problem subject to normal continuity constraints,

$$
\mathbf{u}=\arg \min _{\substack{\mathbf{v} \in \mathcal{S} \\ \text { s.t. } \llbracket \mathbf{n} \cdot \mathbf{v} \rrbracket=0 \text { on } \Gamma \\ \mathbf{n} \cdot \mathbf{v}=\mathbf{n} \cdot \mathbf{u}_{\mathrm{D}} \text { on } \Gamma_{\mathrm{D}}}} \frac{1}{2} a_{\text {IP }}(\mathbf{v}, \mathbf{v})-l_{\text {IP }}(\mathbf{v})
$$

Note that the terms with pressures are canceled, thanks to the imposed continuity constraints. As usual in constrained minimization problems, the previous optimization problem can be solved using a non-consistent penalty, see, for instance, [18]. The corresponding minimization problem with penalty is

$$
\mathbf{u}=\arg \min _{\mathbf{v} \in \mathcal{S}} \frac{1}{2} a_{\mathrm{IP}}(\mathbf{v}, \mathbf{v})-l_{\mathrm{IP}}(\mathbf{v})+\beta\left[(\llbracket \mathbf{n} \cdot \mathbf{v} \rrbracket, \llbracket \mathbf{n} \cdot \mathbf{v} \rrbracket)_{\Gamma}-\left(\mathbf{n} \cdot\left(\mathbf{u}_{\mathrm{D}}-\mathbf{v}\right), \mathbf{n} \cdot\left(\mathbf{u}_{\mathrm{D}}-\mathbf{v}\right)\right)_{\Gamma_{\mathrm{D}}}\right]
$$

where $\beta$ is a scalar penalty to be chosen. The solution of this optimization problem is the solution of the following IPM weak formulation with penalty: find $\mathbf{u}_{\beta} \in \mathcal{S}$ such that

$$
a_{\text {IP }}\left(\mathbf{u}_{\beta}, \mathbf{v}\right)+\beta\left(\llbracket \mathbf{n} \cdot \mathbf{u}_{\beta} \rrbracket, \llbracket \mathbf{n} \cdot \mathbf{v} \rrbracket\right)_{\Gamma \cup \Gamma_{\mathrm{D}}}=l_{\text {IP }}(\mathbf{v})+\beta\left(\mathbf{n} \cdot \mathbf{v}, \mathbf{n} \cdot \mathbf{u}_{\mathrm{D}}\right)_{\Gamma_{\mathrm{D}}}
$$

for all $\mathbf{v} \in \mathcal{S}$. In the following, we refer to this weak formulation as interior penalty method with penalty (IPMP) in front of the IPM formulation described in (14).

Once the velocity is obtained, pressure can be computed as a post-process with two steps. First, an approximation of the hybrid pressure can be obtained introducing the solution of (21) in (14a), namely

$$
\hat{p}_{\beta}= \begin{cases}\beta \llbracket \mathbf{n} \cdot \mathbf{u}_{\beta} \rrbracket & \text { on } \Gamma \\ \beta \mathbf{n} \cdot\left[\mathbf{u}_{\beta}-\mathbf{u}_{\mathrm{D}}\right] & \text { on } \Gamma_{\mathrm{D}}\end{cases}
$$

Then, with $\mathbf{u}_{\beta}$ and $\hat{p}_{\beta}$ the interior pressure can be determined as the solution of (14b).

It is important to remark that the IPMP formulation (21) involves two different penalties with important differences. The first one is inherited from the IPM formulation, i.e. $\gamma / l_{\mathrm{e}}$ in the bilinear form $a_{\mathrm{IP}}(\cdot, \cdot)$ defined in (11a). It is a consistent penalty in the sense that the solution of the original problem (1) is the solution of the IPM formulation (14a); therefore, as usual in IPM formulations, in practice moderate values of the constant parameter $\gamma$ provide accurate and optimally convergent results. This is not the case for the second penalty. The penalty $\beta$ in the IPMP formulation (21) is a non-consistent penalty: the solution of the IPMP formulation verifies the continuity of the normal component of the velocity and the Dirichlet boundary conditions only in the limit, for $\beta$ going to infinity. This lack of consistency is the origin of the usual drawbacks of penalty 
DG METHODS FOR THE STOKES EQUATIONS

techniques: the tuning of the penalty parameter affects the accuracy of the solution and, in practice, too large values of $\beta$ are needed, leading to ill-conditioned systems of equations. In fact, as proved in [18] in the context of boundary conditions, and as it is seen in the numerical examples, the penalty parameter $\beta$ has to be of order $h^{-k}$ in order to keep the optimal $\mathcal{H}^{1}$ convergence rate, with $h$ the element size and $k$ the degree of the approximation.

It is worth noting that an alternative and consistent methodology for the solution of the constrained minimization problem (20) would be the introduction of a Lagrange multiplier. That is,

$$
(\mathbf{u}, \lambda)=\arg \min _{\mathbf{v} \in \mathcal{S}} \max _{\lambda \in \Lambda} \frac{1}{2} a_{\mathrm{IP}}(\mathbf{v}, \mathbf{v})-l_{\mathrm{IP}}(\mathbf{v})+(\lambda, \llbracket \mathbf{n} \cdot \mathbf{v} \rrbracket)_{\Gamma}-\left(\lambda, \mathbf{n} \cdot\left(\mathbf{v}-\mathbf{u}_{\mathrm{D}}\right)\right)_{\Gamma_{\mathrm{D}}}
$$

where $\lambda$ is the Lagrange multiplier defined at $\Gamma \cup \Gamma_{\mathrm{D}}$. This formulation corresponds exactly to (19), or equivalently to the IPM formulation (14a), demonstrating that the hybrid pressure plays the role of a Lagrange multiplier to impose the continuity of the normal velocity.

\section{FINITE-DIMENSIONAL SPACES}

In practice, approximations to the exact solution are obtained using finite-dimensional spaces. In particular, standard finite-dimensional polynomial spaces may be introduced in each element (standard DG) for all the previously defined weak problems, namely

$$
\mathcal{V}^{h}:=\left\{\mathbf{v} \in\left[\mathcal{H}^{1}(\widehat{\Omega})\right]^{\mathrm{n}_{\mathrm{sd}}}|\mathbf{v}|_{\Omega_{i}} \in\left[\mathbb{P}^{k}\left(\Omega_{i}\right)\right]^{\mathrm{n}_{\mathrm{sd}}} \text { for } i=1, \ldots, \mathrm{n}_{\mathrm{el}}\right\}
$$

and

$$
\mathcal{Q}^{h}:=\left\{p \in \mathcal{L}_{2}(\Omega)|p|_{\Omega_{i}} \in \mathbb{P}^{k-1}\left(\Omega_{i}\right) \text { for } i=1, \ldots, \mathrm{n}_{\mathrm{el}}\right\}
$$

where $\mathbb{P}^{m}$ denotes the space of complete polynomials of degree less than or equal to $m$. The finite counterparts of $\mathcal{S}$ and $\mathcal{I}$ are

$$
\begin{aligned}
& \mathcal{S}^{h}=\left\{\mathbf{v} \in\left[\mathcal{H}^{1}(\widehat{\Omega})\right]^{\mathrm{n}_{\mathrm{sd}}}|\mathbf{v}|_{\Omega_{i}} \in\left[\mathbb{P}^{k}\left(\Omega_{i}\right)\right]^{\mathrm{n}_{\mathrm{sd}}},\left.\quad \nabla \cdot \mathbf{v}\right|_{\Omega_{i}}=0 \text { for } i=1, \ldots, \mathrm{n}_{\mathrm{el}}\right\} \\
& \mathcal{I}^{h} \subset\left\{\mathbf{v} \in\left[\mathcal{H}^{1}(\widehat{\Omega})\right]^{\mathrm{n}_{\mathrm{sd}}}|\mathbf{v}|_{\Omega_{i}} \in\left[\mathbb{P}^{k}\left(\Omega_{i}\right)\right]^{\mathrm{n}_{\mathrm{sd}}}, \quad \nabla \times\left.\mathbf{v}\right|_{\Omega_{i}}=\mathbf{0} \text { for } i=1, \ldots, \mathrm{n}_{\mathrm{el}}\right\}
\end{aligned}
$$

such that $\mathcal{I}^{h} \subset \mathcal{I}$. Note that the following relations and inclusions are verified: $\mathcal{V}^{h}=\mathcal{S}^{h} \oplus \mathcal{I}^{h}$, $\mathcal{V}^{h} \subset\left[\mathcal{H}^{1}(\widehat{\Omega})\right]^{\mathrm{n}_{\mathrm{sd}}}, \mathcal{Q}^{h} \subset \mathcal{L}_{2}(\Omega)$ and $\mathcal{S}^{h} \subset \mathcal{S}$. The finite-dimensional subspace associated with the hybrid pressures, $\mathcal{P}^{h} \subset \mathcal{P}$, can be defined directly from (13) restricting velocities to $\mathcal{S}^{h}$. In fact, Reference [6] also demonstrates that $\mathcal{P}^{h}$ corresponds to piecewise polynomial pressures in the element edges in 2D or faces in 3D.

It is worth noting that the definition of the solenoidal and irrotational polynomial bases to be used at each element is an easy task. For instance, a solenoidal base in a $2 \mathrm{D}$ triangle for an approximation of degree $k=2$ is

$$
\mathcal{S}^{h}=\left\langle\left(\begin{array}{l}
1 \\
0
\end{array}\right),\left(\begin{array}{l}
0 \\
1
\end{array}\right),\left(\begin{array}{l}
0 \\
x
\end{array}\right),\left(\begin{array}{c}
x \\
-y
\end{array}\right),\left(\begin{array}{l}
y \\
0
\end{array}\right),\left(\begin{array}{c}
0 \\
x^{2}
\end{array}\right),\left(\begin{array}{c}
2 x y \\
-y^{2}
\end{array}\right),\left(\begin{array}{c}
x^{2} \\
-2 x y
\end{array}\right),\left(\begin{array}{l}
y^{2} \\
0
\end{array}\right)\right\rangle
$$


The use of this polynomial basis defined with Cartesian coordinates was also proposed in [6]. An irrotational base for $k=2$ is

$$
\mathcal{I}^{h}=\left\langle\left(\begin{array}{l}
x \\
0
\end{array}\right),\left(\begin{array}{l}
x^{2} \\
0
\end{array}\right),\left(\begin{array}{c}
0 \\
y^{2}
\end{array}\right)\right\rangle
$$

In the numerical examples, to avoid ill-conditioning of the elemental matrices, all polynomials $p$ of the base are centered and scaled at each element as $p\left(\left(\mathbf{x}-\mathbf{c}_{\mathrm{e}}\right) / h_{\mathrm{e}}\right)$, where $\mathbf{c}_{\mathrm{e}}$ and $h_{\mathrm{e}}$ denote the center and the size of the element, respectively.

\section{Remark 2}

With these polynomial spaces, the numerical solution $\mathbf{u}_{h}$ of the IPM method presented in Section 3.2 verifies the following error bound:

$$
\left\|\left|\mathbf{u}-\mathbf{u}_{h}\right|\right\| \leqslant K h^{\alpha}|\mathbf{u}|_{\mathcal{H}^{1+\alpha}(\Omega)}
$$

for $\mathbf{u} \in \mathcal{H}^{1+\alpha}(\Omega), 1 \leqslant \alpha \leqslant k$ and some constant $K$. This result can be proved using the continuity and coercivity of the bilinear form, see Remark 1 in Section 3.2. Following [9], the space of piecewise divergence-free polynomial functions with continuity constraints for the normal velocity is considered

$$
Z_{h}\left(\mathbf{u}_{\mathrm{D}}\right)=\left\{\mathbf{v} \in \mathcal{S}^{h}:(q, \llbracket \mathbf{n} \cdot \mathbf{v} \rrbracket) \Gamma_{\Gamma} \Gamma_{\mathrm{D}}=\left(q, \mathbf{n} \cdot \mathbf{u}_{\mathrm{D}}\right)_{\Gamma_{\mathrm{D}}} \forall q \in \mathcal{P}^{h}\right\} \subset \mathcal{S}^{h}
$$

Note that although the LDG formulation analyzed in [9] verifies an approximate orthogonality with a residual $\mathcal{R}_{h} \neq 0$ (due to the introduction of the lifting operators), the IPM formulation is consistent and therefore the residual is in this case $\mathcal{R}_{h}=0$. Thus, the particularization of the error bound stated in [9] is

$$
\left\|\mathbf{u}-\mathbf{u}_{h}\right\| \leqslant(1+m) \inf _{\mathbf{v} \in Z_{h}\left(\mathbf{u}_{\mathrm{D}}\right)}\|\mathbf{u}-\mathbf{v}\|
$$

where $m$ is the coercivity constant, see Remark 1 . The error bound (22) is obtained considering the projection into the $\mathrm{BDM}_{0}$ space (Brezzi-Douglas-Marini space of full polynomial approximations with normal continuity and zero elementwise divergence, see [19] for details), that is $\mathbf{v}=\pi^{\mathrm{BDM}} \mathbf{u}$. Note that $\mathrm{BDM}_{0} \subset Z_{h}\left(\mathbf{u}_{\mathrm{D}}\right)$, thus using the bound in [13] for the $\||\cdot \||$ norm, i.e.

$$
\left\|\left.\left|\mathbf{u}-\pi^{\mathrm{BDM}} \mathbf{u} \| \leqslant C h^{\alpha}\right| u\right|_{\mathcal{H}^{1+\alpha}(\Omega)}\right.
$$

with some constant $C$, bound (22) is proved.

\section{Remark 3}

The convergence of the IPMP formulation, developed in Section 3.3, is analyzed in detail in [13] for different approximation spaces. For velocity approximation spaces including the $\mathrm{BDM}_{0} \mathrm{space}_{\text {, }}$ the error bound is

$$
\left\|\mathbf{u}-\mathbf{u}_{h}\right\| \mid \leqslant C\left(h^{\alpha}|\mathbf{u}|_{\mathcal{H}^{1+\alpha}(\Omega)}+h\|p\|_{\mathcal{H}^{1}(\Omega)}\right)
$$

for some constant $C$, and $\mathbf{u} \in \mathcal{H}^{1+\alpha}(\Omega)$, with $1 \leqslant \alpha \leqslant k$. 


\section{NUMERICAL EXAMPLES}

To demonstrate the applicability of the two proposed methods, some numerical examples are shown in this section. In all tests, an approximation of order $k$ for velocity and $k-1$ for pressure is considered. Triangular meshes are obtained by splitting a regular $n \times m$ Cartesian grid into a total of $2 n \times m$ triangles for a rectangular domain, or $2 n^{2}$ triangles for a square domain, giving uniform element size of $h=1 / n$.

\subsection{Driven cavity example}

A standard benchmark test for incompressible flows is considered first. A plane flow of an isothermal fluid in a lid-driven cavity is modeled in a $2 \mathrm{D}$ square domain $\Omega=] 0,1[\times] 0,1$, with zero body force and one moving wall. A velocity $\mathbf{u}=(1,0)^{\mathrm{T}}$ is imposed on the exterior upper boundary $\{y=1\}$, and a zero velocity $\mathbf{u}=(0,0)^{\mathrm{T}}$ is enforced on the other three sides.

Figure 1 shows the velocity vectors and the pressure fields of the flow for, respectively, the IPM and the IPMP formulations, with a discretization of order $k=2$ for velocity and order $k-1=1$ for pressure. Results fit to the expected solution; note that around the two upper corners the pressure

(a)
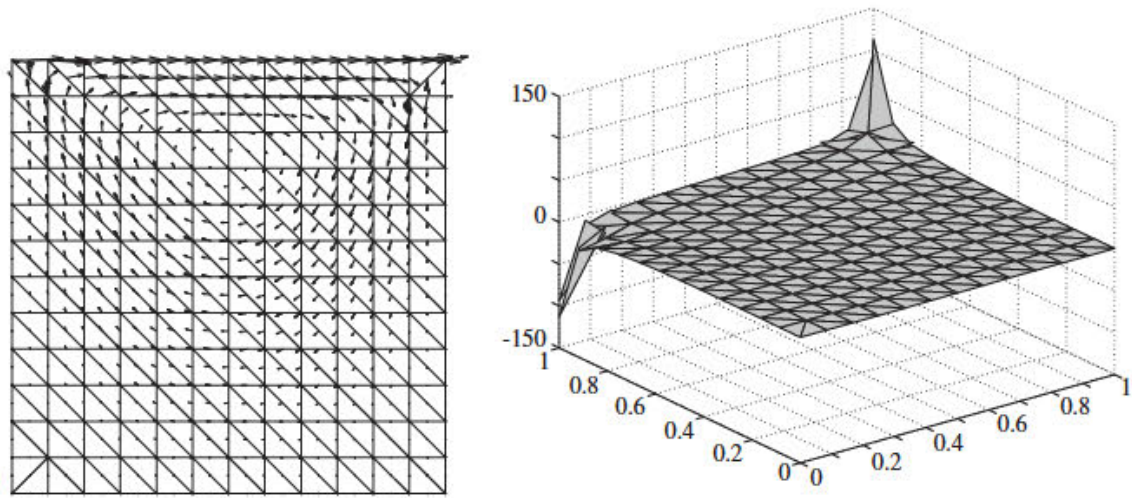

(b)
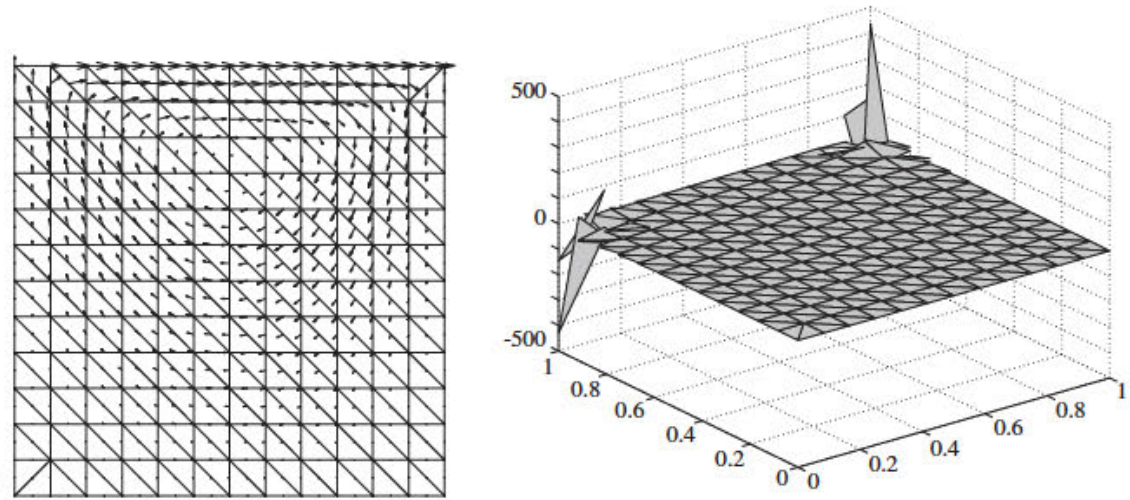

Figure 1. Driven cavity IPM (top) and IPMP (bottom) results for second-order velocity and linear pressure: (a) IPM velocity and pressure with $\gamma=10$ and (b) IPMP velocity and pressure with $\gamma=10$ and $\beta=1000 / h^{2}$. 

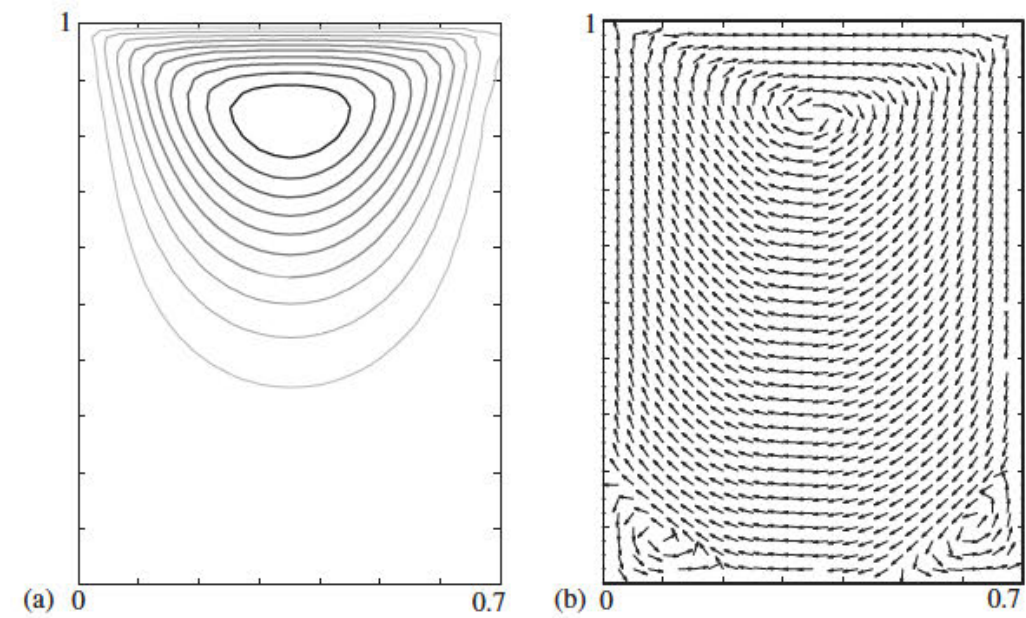

Figure 2. IPM velocity solution with 140 elements, fourth-order velocity approximation and $\gamma=20$ : (a) velocity streamlines and (b) scaled velocity.

takes not bounded values because of the discontinuity of the velocity. Recall that the computation of velocity and pressure is completely decoupled using the IPMP, with the corresponding saving in computational cost. Nevertheless, it is worth noting that the use of the non-consistent parameter $\beta=1000 / h^{2}$ in the IPMP considerably increases the condition number of the matrix. Moreover, although similar accuracy is obtained for the velocity field, for the same discretization the IPM provides more accurate and stable results for the pressure field than the IPMP.

The same example is now used for a rectangular cavity $\Omega=] 0,0.7[\times] 0,1[$. Figure 2 illustrates the results obtained using the IPM formulation. The results present the expected behavior. Contrarotating vortices are created in the corners opposite to the moving wall. In the representation of the velocity vectors, only the direction of the flow is represented, all the arrows have the same length so that the contra-rotating vortices can be noticed. The velocity streamlines are represented as well to prove that the contra-rotating vortices have small amplitude compared with the main vortex movement.

\subsection{Analytical example}

An example with analytical solution is now considered to study the accuracy and convergence properties of the proposed methodologies. The Stokes equations are solved in a 2D square domain $\Omega=] 0,1[\times] 0,1[$ with Dirichlet boundary conditions on three edges, and a Neumann boundary condition on the fourth edge $\{y=0\}$. A body force

$$
\mathbf{f}=\left(\begin{array}{c}
12(1-2 y) x^{4}+24(-1+2 y) x^{3}+12\left(-4 y+6 y^{2}-4 y^{3}+1\right) x^{2} \\
+\left(-2+24\left(y-3 y^{2}+2 y^{3}\right)\right) x+1-4 y+12 y^{2}-8 y^{3} \\
8\left(1-6 y+6 y^{2}\right) x^{3}+12\left(-1+6 y-6 y^{2}\right) x^{2} \\
+\left(4+48\left(y^{2}-y^{3}\right)+24\left(y^{4}-y\right)\right) x-12 y^{2}+24 y^{3}-12 y^{4}
\end{array}\right)
$$


is imposed in order to have the polynomial exact solution

$$
\begin{gathered}
\mathbf{u}=\left(\begin{array}{c}
x^{2}(1-x)^{2}\left(2 y-6 y^{2}+4 y^{3}\right) \\
-y^{2}(1-y)^{2}\left(2 x-6 x^{2}+4 x^{3}\right)
\end{array}\right) \\
p=x(1-x)
\end{gathered}
$$

5.2.1. IPM analysis. The behavior of the IPM formulation is first studied. In all examples, the consistent penalty term $\gamma$ is set to a sufficiently large value to ensure the coercivity of the form $a_{\mathrm{IP}}($,$) , see Equation (11a). In practice, moderate values of this penalty term are required.$

Figure 3 shows the IPM solution obtained with an approximation of degree $k=2$ and 4 for the velocity field ( $k-1$ for pressure), with the same number of degrees of freedom. One of the advantages of the proposed method is that the order of the approximation can be easily increased, with a straightforward modification of the definition of the solenoidal and irrotational bases, see Section 4. As expected, the higher-order approximation provides more accurate results, with smaller discontinuities in the solution, especially for the pressure field.

(a)
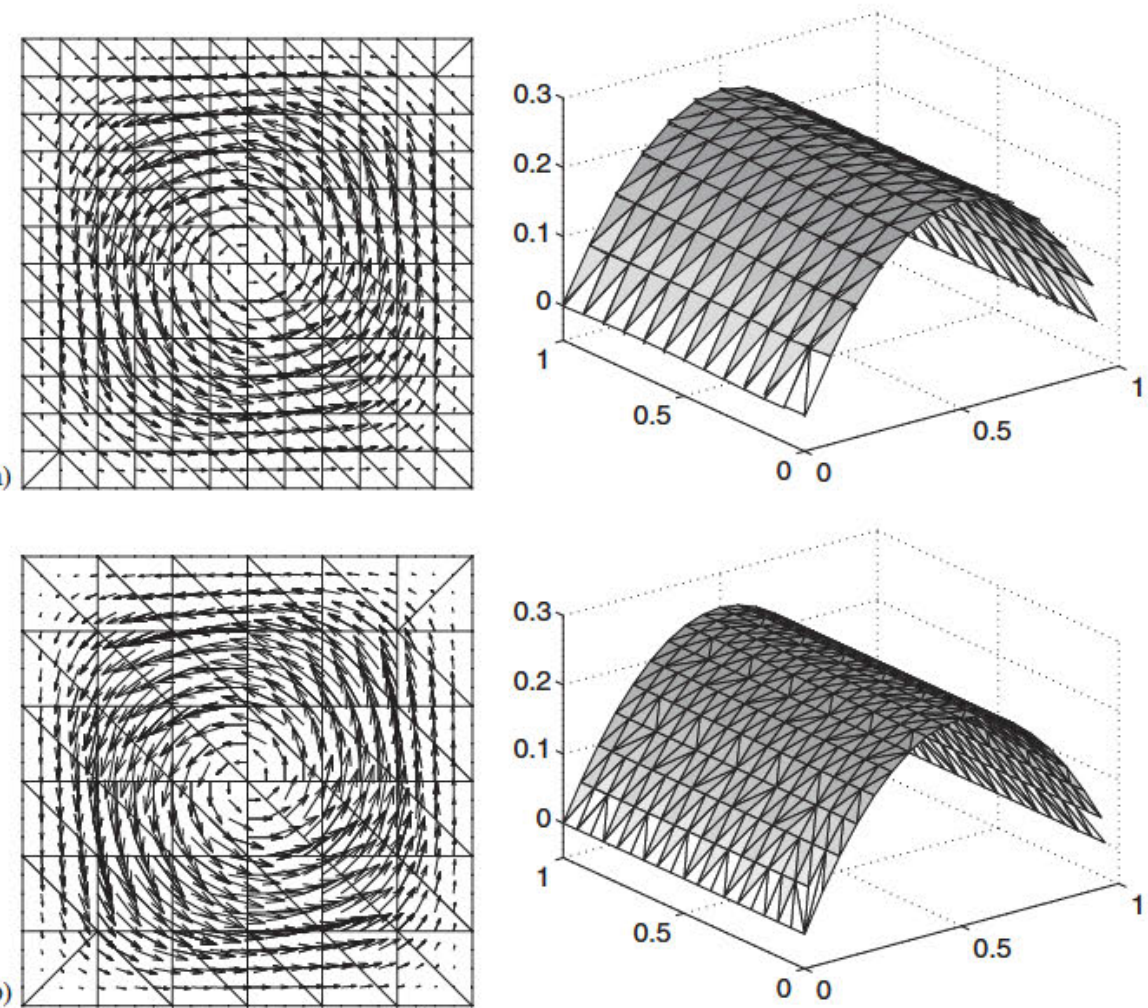

Figure 3. IPM velocity vectors and pressure field for two different orders of approximation: (a) velocity and pressure with degree $k=2,256$ elements and $\gamma=10$ and (b) velocity and pressure with degree $k=4,72$ elements and $\gamma=40$. 
A. MONTLAUR, S. FERNANDEZ-MENDEZ AND A. HUERTA
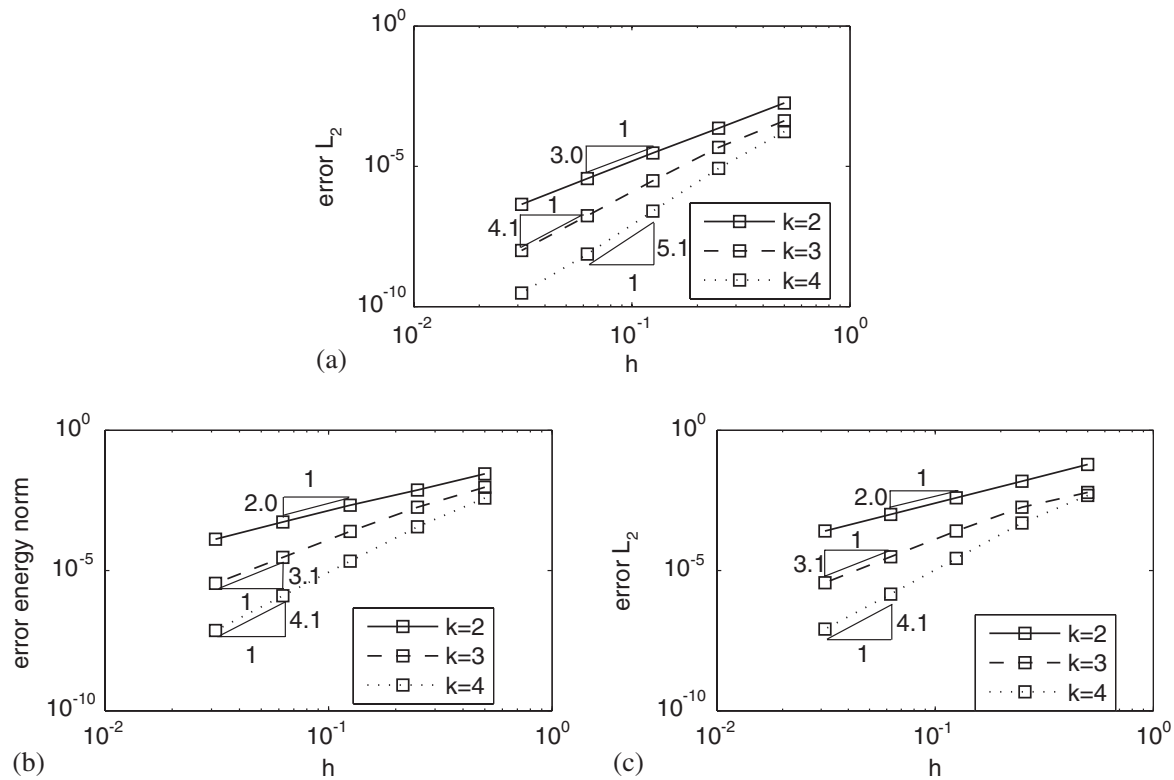

Figure 4. IPM convergence results with velocity approximation of degree $k=2,3,4$ and pressure interpolation of degree $k-1$, with $\gamma=10,20,40$, respectively: (a) velocity $\mathcal{L}_{2}$ error; (b) velocity energy error; and (c) pressure $\mathcal{L}_{2}$ error.

These results also confirm that the condition proposed in [20] to ensure the coercivity of the bilinear form is also valid for the IPM formulation with solenoidal approximation proposed here. The explicit formula used for the computation of the consistent penalty parameter is

$$
\gamma \approx a v k^{2}
$$

where $a$ is a positive constant and $k$ is the degree of the velocity approximation.

Figure 4 shows the convergence under $h$-refinement, for different orders of approximation of the velocity and pressure. Optimal convergence is obtained using polynomials of degree $k$ to approximate the velocity and $k-1$ for pressure; that is, convergence of order $k+1$ for the velocity $\mathcal{L}_{2}$ norm, order $k$ for the energy norm, and order of $k$ for the pressure $\mathcal{L}_{2}$ norm. As usual in consistent IPM formulations, a penalty term of order $h^{-1}$, i.e constant $\gamma$, suffices to maintain the optimal convergence rates for any order of approximation. As seen in the following examples, this is not the case for the non-consistent penalty $\beta$ in the IPMP formulation.

5.2.2. IPMP analysis. The IPMP behavior is tested with the same analytical example. First, the influence of the non-consistent penalty term $\beta$ is analyzed. The IPMP velocity for an approximation of degree $k=3$, with two different values of the non-consistent penalty parameter $\beta$ is depicted in Figure 5. As previously commented, rather large values of $\beta$ are necessary to ensure moderate discontinuities of the normal velocity.

Figure 6 shows the results for two different orders of approximation. Again, higher-order approximations provide more accurate results for the same number of degrees of freedom, especially for the pressure field that presents much better continuity. 

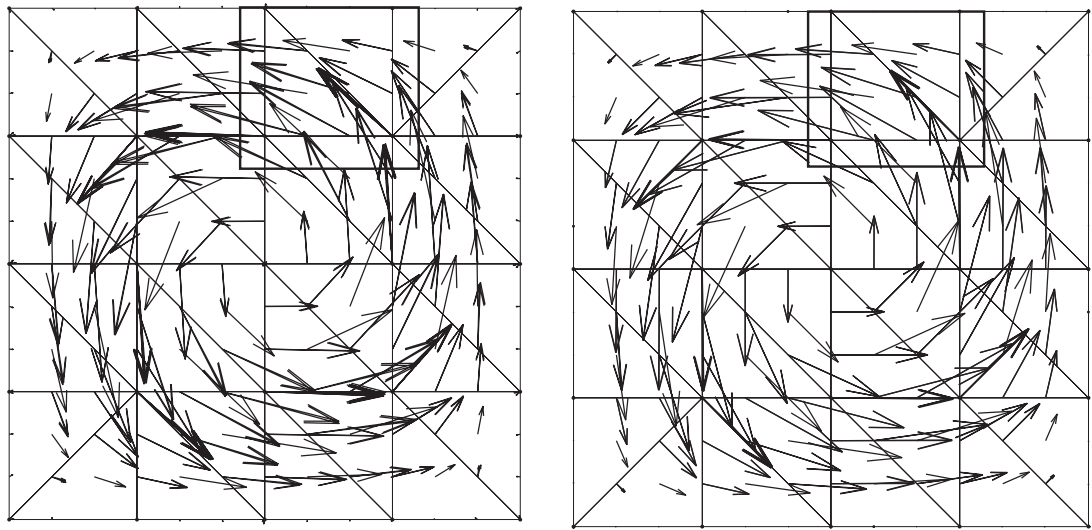

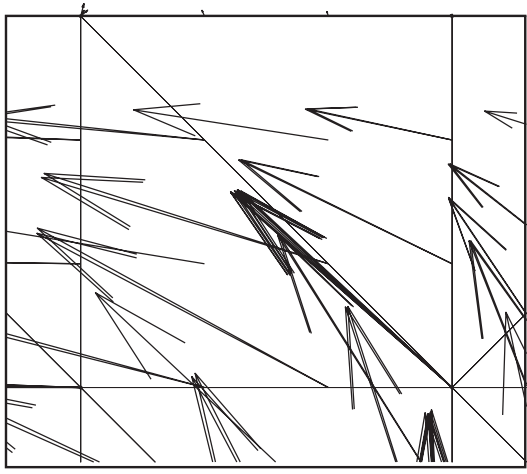

(a)

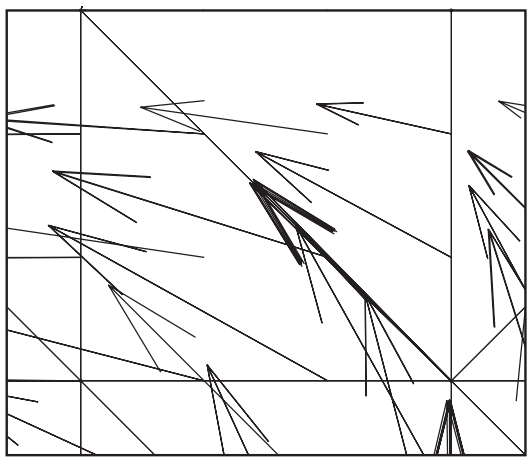

(b)

Figure 5. IPMP velocity solution (top) and detail (bottom) for two different values of the penalty term $\beta$ with a third-order velocity approximation and 32 elements: (a) $\beta=5 / h^{4}$ and (b) $\beta=2000 / h^{4}$.

Figure 7 shows the evolution of the error under $h$-refinement for different orders of approximation of the velocity and pressure, using the IPMP formulation. As usual for non-consistent penalty formulations [18], almost optimal converge rates are achieved using a penalty term of order $h^{-k}$. As previously noted, the need of large values for the penalty $\beta$ is the main drawback of the IPMP formulation, because of the ill-conditioning of the matrices in the solution with fine meshes. For instance, for a computation with fourth-order interpolation of the velocity and 72 elements, the dimension of the system of equations to be solved for the IPM (with velocity and hybrid pressures) is 1350 , whereas for the IPMP (with only velocities) the dimension is 1308 . The reduction in the number of degrees of freedom is thus appreciable for the IPMP case, but in return the condition number of the matrix is higher for the IPMP formulation: around $5 \times 10^{9}$ for the IPMP with $\gamma=40$, and $4 \times 10^{7}$ for the IPM with the same $\gamma$ and $\beta=4000 / h^{4}$. Moreover, under $h$-refinement or $p$-refinement, the condition number grows faster for the IPMP than for the IPM.

To further compare the IPM and IPMP formulations, Figure 8 plots the errors obtained for velocity and pressure with both methods. Similar accuracy is obtained for the velocity field and the main differences are present in the pressure results. Although both methods provide optimal convergence rates, more accurate results for pressure are obtained with a coupled computation 
(a)
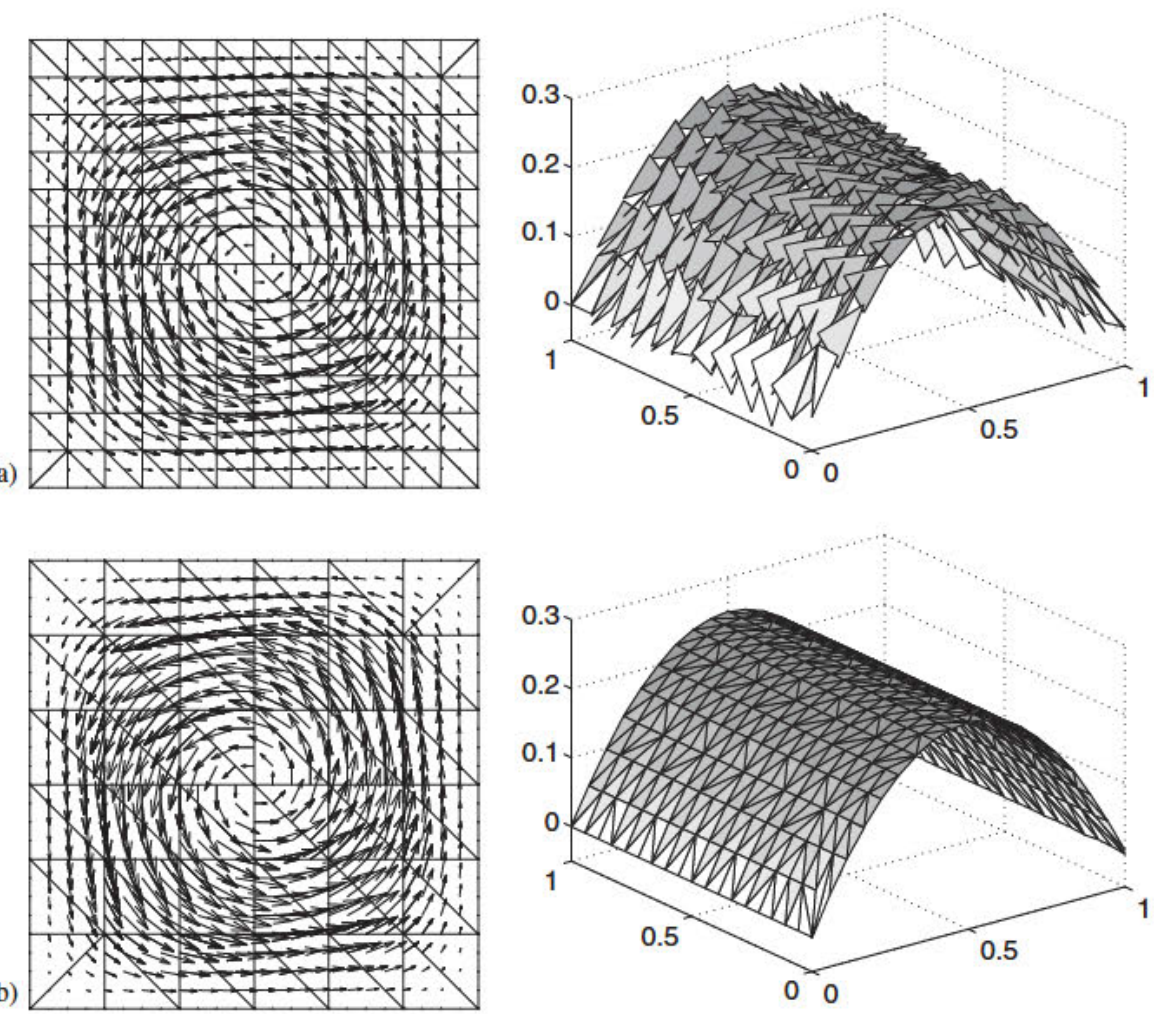

Figure 6. IPMP velocity vectors and pressure field for different orders of approximation: (a) velocity and pressure with degree $k=2,256$ elements, $\gamma=10$ and $\beta=1000 / h^{2}$ and (b) velocity and pressure with degree $k=4,72$ elements, $\gamma=40$ and $\beta=4000 / h^{4}$.

of hybrid pressures and velocities, using the IPM formulation. As commented in the previous example in Section 5.2, the computation of pressures as a post-process of velocities with the IPMP represents a saving in computational cost, preserving the accuracy in the velocity field, but with a slightly worse solution for pressure.

\subsection{Flow in an idealized porous medium}

A fluid in an idealized porous medium is subject to a friction force proportional to the fluid velocity u. This kind of problem is derived from the Stokes equations and it follows Darcy's law. It is valid for slow, viscous flow, such as groundwater flows. The problem to be solved is

$$
\begin{aligned}
-\nabla \cdot \boldsymbol{\sigma} & =-\alpha \mathbf{u} & & \text { in } \widehat{\mathbf{\Omega}} \\
\boldsymbol{\nabla} \cdot \mathbf{u} & =0 & & \text { in } \widehat{\Omega} \\
\mathbf{u} & =\mathbf{u}_{\mathrm{D}} & & \text { on } \Gamma_{\mathrm{D}} \\
\llbracket \mathbf{n} \otimes \mathbf{u} \rrbracket & =\mathbf{0} & & \text { on } \Gamma \\
\llbracket \mathbf{n} \cdot \boldsymbol{\sigma} \rrbracket & =\mathbf{0} & & \text { on } \Gamma
\end{aligned}
$$


DG METHODS FOR THE STOKES EQUATIONS
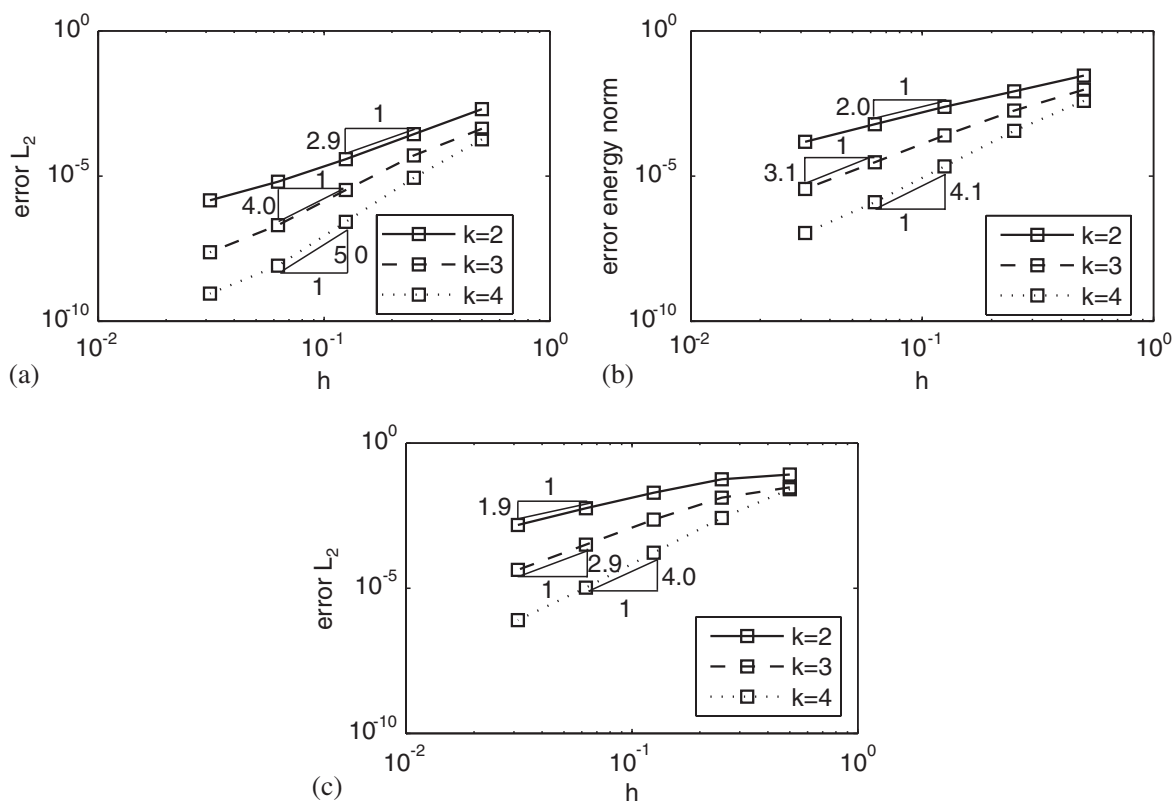

Figure 7. IPMP convergence results with degree $k=2,3,4$ for velocity and degree $k-1$ for pressure, with $\gamma=10,20,40$ and $\beta=1000 / h^{2}, 2000 / h^{3}, 4000 / h^{4}$, respectively: (a) velocity $\mathcal{L}_{2}$ error; (b) velocity energy error; and (c) pressure $\mathcal{L}_{2}$ error.
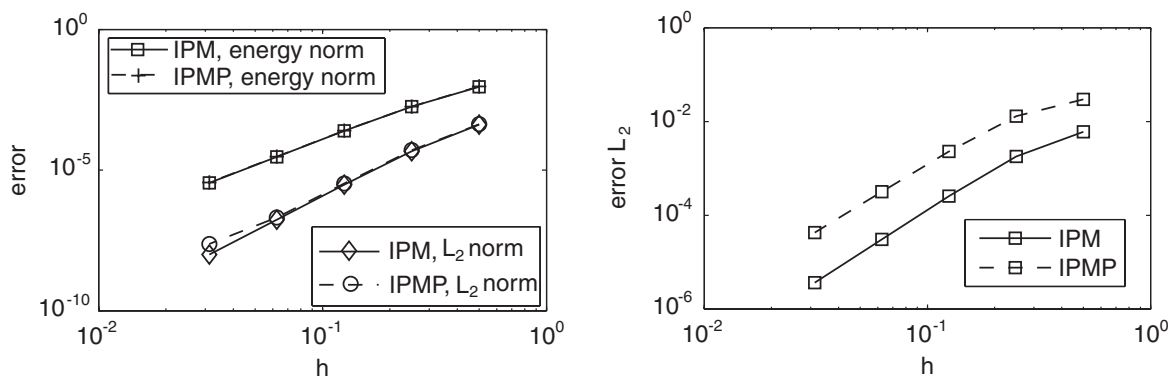

Figure 8. Comparison of the errors obtained with IPM and IPMP, for a cubic approximation of the velocity (left) and a quadratic interpolation of the pressure (right), with $\gamma=20$ and $\beta=2000 / h^{3}$.

where $\alpha$ is the inverse of the local permeability of the medium ( $\alpha=0$ for an empty medium and $\alpha=+\infty$ for a solid wall), see [21].

These equations are solved in the computational domain shown in Figure 9, consisting of a long straight channel of height $l$ and length $L=10 l$. The porous domain is limited to the central part of length $5 l$. The Dirichlet boundary conditions prescribe a parabolic velocity profile at the inlet and at the outlet, and a no-slip condition for the fluid on the channel side. The porous domain is filled 


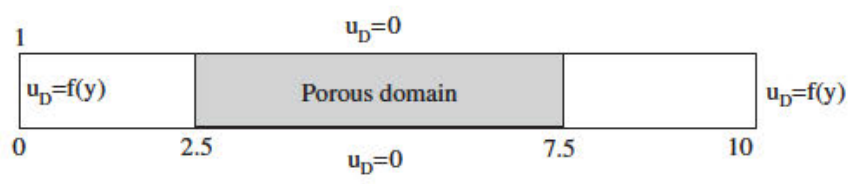

Figure 9. Computational domain. The porous domain is limited to the central part, of length $5 l$ and height $l$.

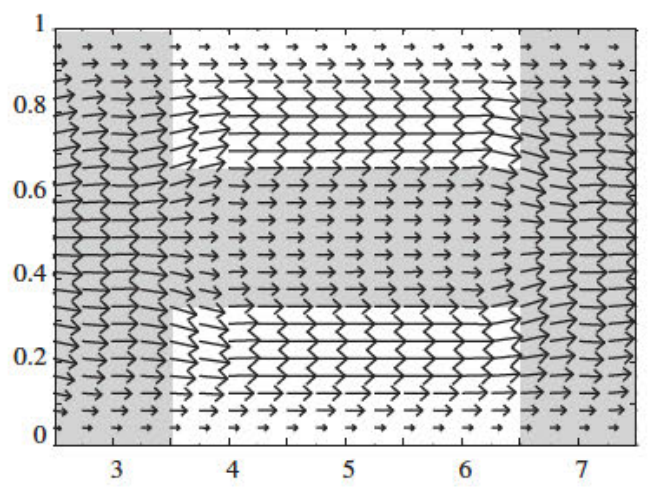

Figure 10 . Velocity vectors within the porous domain of length $5 l$. The grey part represents a porous material, the white ones an empty domain.

with porous material of arbitrary value $\alpha=100$ for $2.5<x<7.5$ except for two regions verifying

$$
x \in] 3.5,6.5[\text { and } y \in] 0, \frac{1}{3}[\cup] \frac{2}{3}, 1[
$$

where empty medium is assumed, see white region in Figure 10.

Details of the IPM velocity result in the porous domain are shown in Figure 10, demonstrating the capability of the IPM formulation for the solution of these problem types. As expected, the two empty regions divert the flow away from the center of the channel: the flow tends to go into the empty domains, with higher velocities than the porous region.

\section{CONCLUDING REMARKS}

Two discontinuous Galerkin (DG) formulations with solenoidal approximation for the simulation of incompressible flow are proposed, with application to the Stokes equation. Following the methodology of the interior penalty method (IPM), and considering a solenoidal and irrotational decomposition of the interpolation space, an efficient DG formulation for the computation of velocities and hybrid pressures (pressures along the element sides) is developed. Moreover, the introduction of a penalty parameter for the weak enforcement of continuity of the normal velocity along element sides leads to an alternative DG formulation where the computation of velocities and pressures is completely decoupled. This second formulation coincides with the formulation 


\section{DG METHODS FOR THE STOKES EQUATIONS}

proposed in [13] and allows to compute the velocity field with no presence of pressure terms; the pressure field can then be obtained as a post-process of the velocity solution.

Numerical experiments demonstrate the applicability of the proposed methods, with optimal convergence rates under $h$-refinement. The effect of the penalty parameter is also analyzed: as usual in IPM formulations, a penalty of order $h^{-1}$ provides optimal results, whereas the non-consistent penalty in the second formulation must be of order $h^{-k}$, with $k$ the degree of the approximation. Thus, for large engineering computations this second formulation represents an important save in the number of degrees of freedom in front of the IPM or alternative formulations, but as usual in non-consistent penalty formulations, it may lead to ill-conditioned systems of equations. Moreover, for the same discretization the IPM provides more accurate pressure results than the second formulation.

\section{REFERENCES}

1. Crouzeix M, Raviart P-A. Conforming and nonconforming finite element methods for solving the stationary Stokes equations. I. Revue Française d'Automatique Informatique Recherche Opérationnelle, Série Rouge 1973; 7(R-3):33-75.

2. Griffiths DF. An approximately divergence-free 9-node velocity element (with variations) for incompressible flows. International Journal for Numerical Methods in Fluids 1981; 1(4):323-346.

3. Gunzburger MD. Finite Element Methods for Viscous Incompressible Flows. A Guide to Theory, Practice, and Algorithms. Academic Press: Boston, MA, 1989.

4. Cockburn B. Discontinuous Galerkin methods for computational fluid dynamics. In Encyclopedia of Computational Mechanics, vol. 3. Fluids, Chapter 4, Stein E, de Borst R, Hughes TJR (eds). Wiley: Chichester, 2004; 91-127.

5. Baker GA, Jureidini WN, Karakashian OA. Piecewise solenoidal vector fields and the Stokes problem. SIAM Journal on Numerical Analysis 1990; 27(6):1466-1485.

6. Cockburn B, Gopalakrishnan J. Incompressible finite elements via hybridization. Part I: The Stokes system in two space dimensions. SIAM Journal on Numerical Analysis 2005; 43(4):1627-1650.

7. Toselli A. $h p$ discontinuous Galerkin approximations for the Stokes problem. Mathematical Models and Methods in Applied Sciences 2002; 12(11):1565-1597.

8. Karakashian OA, Jureidini WN. A nonconforming finite element method for the stationary Navier-Stokes equations. SIAM Journal on Numerical Analysis 1998; 35(1):93-120.

9. Carrero J, Cockburn B, Schötzau D. Hybridized globally divergence-free LDG methods. Part I: The Stokes problem. Mathematics of Computation 2006; 75:533-563.

10. Cockburn B, Kanschat G, Schötzau D. A note on discontinuous Galerkin divergence-free solutions of the Navier-Stokes equations. Journal on Scientific Computing 2007; 31(1-2):61-73.

11. Arnold DN. An interior penalty finite element method with discontinuous elements. SIAM Journal on Numerical Analysis 1982; 19(4):742-760.

12. Hansbo A, Hansbo P. A finite element method for the simulation of strong and weak discontinuities in solid mechanics. Computer Methods in Applied Mechanics and Engineering 2004; 193(33-35):3523-3540.

13. Hansbo P, Larson MG. Piecewise divergence-free discontinuous Galerkin methods for stokes flow. Communications in Numerical Methods in Engineering 2006; DOI: 10.1002/cnm.975.

14. Hansbo P, Larson MG. Discontinuous Galerkin methods for incompressible and nearly incompressible elasticity by Nitsche's method. Computer Methods in Applied Mechanics and Engineering 2002; 191(17-18):18951908.

15. Hansbo A, Hansbo P. An unfitted finite element method, based on Nitsche's method, for elliptic interface problems. Computer Methods in Applied Mechanics and Engineering 2002; 191(47-48):5537-5552.

16. Mozolevsky I, Süri E, Bösing PR. Discontinuous Galerkin finite element approximations of the two-dimensional Navier-Stokes equations in stream-function formulation. Communications in Numerical Methods in Engineering 2007; 23(6):447-459.

17. Arnold DN, Brezzi F, Cockburn B, Marini LD. Unified analysis of discontinuous Galerkin methods for elliptic problems. SIAM Journal on Numerical Analysis 2002; 39(5):1749-1779.

18. Babuska I. The finite element method with penalty. Mathematics of Computation 1973; 27:221-228. 


\section{A. MONTLAUR, S. FERNANDEZ-MENDEZ AND A. HUERTA}

19. Brezzi F, Fortin M. Mixed and Hybrid Finite Element Methods. Springer: Berlin, 1991.

20. Prudhomme S, Pascal F, Oden J, Romkes A. High-order accurate time-stepping schemes for convection-diffusion problems. Technical Report 00-27, TICAM, Austin, TX, 2000.

21. Okkels F, Olesen L, Bruus H. Applications of topology optimization in the design of micro- and nanofluidic systems. Technical Proceedings of the 2005 NSTI Nanotechnology Conference and Trade Show, Anaheim, CA, 2005; 575-578. 